\title{
An extension of MacMahon's Equidistribution Theorem to ordered multiset partitions
}

\author{
Andrew Timothy Wilson $]^{*}$
}

UC San Diego, USA

\begin{abstract}
A classical result of MacMahon states that inversion number and major index have the same distribution over permutations of a given multiset. In this work we prove a strengthening of this theorem originally conjectured by Haglund. Our result can be seen as an equidistribution theorem over the ordered partitions of a multiset into sets, which we call ordered multiset partitions. Our proof is bijective and involves a new generalization of Carlitz's insertion method. As an application, we develop refined Macdonald polynomials for hook shapes. We show that these polynomials are symmetric and give their Schur expansion.
\end{abstract}

Résumé. Un résultat classique de MacMahon affirme que nombre d'inversion et l'indice majeur ont la même distribution sur permutations d'un multi-ensemble donné. Dans ce travail, nous démontrons un renforcement de ce théorème origine conjecturé par Haglund. Notre résultat peut être considéré comme un théorème d'équirépartition sur les partitions ordonnées d'un multi en ensembles, que nous appellerons partitions de multiset commandés. Notre preuve est bijective et implique une nouvelle généralisation de la méthode d'insertion de Carlitz. Comme application, nous développons des polynômes de Macdonald raffinés pour formes d'hameçons. Nous montrons que ces polynômes sont symétriques et donnent leur expansion Schur.

Keywords: inversion number, major index, permutation statistics, insertion method, ordered multiset partitions, Macdonald polynomials

\section{Introduction}

In this section, we fix notation and state our main results. A statistic is a map from a set of objects into the set $\{0,1,2, \ldots\}$. Often our set of objects will be $\mathfrak{S}_{n}$, the group of permutations of $\{1,2, \ldots, n\}$, or, more generally, $\mathfrak{S}_{A}$, the set of permutations of a multiset $A=\left\{1^{a_{1}}, 2^{a_{2}}, \ldots, n^{a_{n}}\right\}$. Given $\sigma \in \mathfrak{S}_{A}$, the descent and ascent sets of $\sigma$ are

$$
\operatorname{Des}(\sigma)=\left\{i: \sigma_{i}>\sigma_{i+1}\right\} \quad \operatorname{Asc}(\sigma)=\left\{i: \sigma_{i}<\sigma_{i+1}\right\}
$$

The inversions of $\sigma$ are the pairs

$$
\operatorname{Inv}(\sigma)=\left\{(i, j): 1 \leq i<j \leq n, \sigma_{i}>\sigma_{j}\right\} .
$$

*atwilsoneucsd.edu. Partially supported by National Defense Science and Engineering Graduate (NDSEG) Fellowship 32 CFR 168a.

1365-8050 @ 2014 Discrete Mathematics and Theoretical Computer Science (DMTCS), Nancy, France 
It will be convenient to refine the set of inversions in the following manner.

$$
\operatorname{Inv}^{i, \square}=\left\{(i, j): i<j \leq n, \sigma_{i}>\sigma_{j}\right\} \quad \operatorname{Inv}^{\square, j}=\left\{(i, j): 1 \leq i<j, \sigma_{i}>\sigma_{j}\right\}
$$

These are the elements of $\operatorname{Inv}(\sigma)$ whose first (resp. second) coordinate is $i$ (resp. $j$ ). These sets allow us to define several statistics on $\mathfrak{S}_{A}$ :

$$
\operatorname{des}(\sigma)=|\operatorname{Des}(\sigma)| \quad \operatorname{asc}(\sigma)=|\operatorname{Asc}(\sigma)| \quad \operatorname{maj}(\sigma)=\sum_{i \in \operatorname{Des}(\sigma)} i \quad \operatorname{inv}(\sigma)=|\operatorname{Inv}(\sigma)|
$$

These statistics are known as the descent number, ascent number, major index, and inversion number of $\sigma$, respectively. We will also make use of two variants of the inversion number:

$$
\operatorname{inv}^{i, \square}(\sigma)=\left|\operatorname{Inv}^{i, \square}(\sigma)\right| \quad \operatorname{inv}^{\square, j}(\sigma)=\left|\operatorname{Inv}^{\square, j}(\sigma)\right| .
$$

Given a statistic stat on a set of objects $\mathrm{Obj}$, the distribution of stat over Obj is the polynomial

$$
D_{\mathrm{Obj}}^{\mathrm{stat}}(q)=\sum_{\sigma \in \mathrm{Obj}} q^{\mathrm{stat}(\sigma)}
$$

Two statistics, say stat on $\mathrm{Obj}$ and stat ${ }^{\prime}$ on $\mathrm{Obj}^{\prime}$, are said to be equidistributed if their distributions are equal. One particular nice way to prove equidistribution is to give a bijection $f: \mathrm{Obj} \rightarrow \mathrm{Obj}^{\prime}$ such that $\operatorname{stat}^{\prime}(f(\sigma))=\operatorname{stat}(\sigma)$ for every $\sigma \in \operatorname{Obj}$. Our main result will be of this form.

In MacMahon (1915), MacMahon showed that the inversion number and major index are equidistributed over $\mathfrak{S}_{A}$, and that $D_{\mathfrak{S}_{A}}^{\text {inv }}(q)=D_{\mathfrak{S}_{A}}^{\text {maj }}(q)=\left[\begin{array}{c}|A| \\ a_{1}, a_{2}, \ldots, a_{n}\end{array}\right]$, where the last expression is the usual $q$-multinomial coefficient. MacMahon's proof was not bijective; the first bijective proof of this fact was given in Foata (1968). A second proof, essentially due to Carlitz (1975), is sometimes known as the insertion method. Our main theorem is a new refinement of the insertion method, which was originally conjectured by Haglund (personal communication via J. B. Remmel, October 2012).

\section{Theorem 1.1}

$$
\sum_{\sigma \in \mathfrak{S}_{A}} q^{\operatorname{inv}(\sigma)} \prod_{i \in \operatorname{Des}(\sigma)}\left(1+\frac{z}{q^{1+\mathrm{inv}^{\square, i}(\sigma)}}\right)=\sum_{\sigma \in \mathfrak{S}_{A}} q^{\operatorname{maj}(\sigma)} \prod_{j=1}^{\operatorname{des}(\sigma)}\left(1+\frac{z}{q^{j}}\right)
$$

When we set $z=0$ in Theorem 1.1 we recover MacMahon's Theorem. Our theorem can be thought of as an extension of MacMahon's Theorem to ordered partitions of multisets into sets, which we will call ordered multiset partitions. Remmel and Wilson (2013) proved the special case of this result where $A=\{1,2, \ldots, n\}$.

If one prefers to deal only with $\mathfrak{S}_{n}$, the technique known as standardization provides a way to relate permutations of multisets to permutations in $\mathfrak{S}_{n}$. Applying standardization to Theorem 1.1. we obtain that the two expressions are equal when summing over those $\sigma \in \mathfrak{S}_{n}$ whose inverse descent sets, i.e. $\operatorname{Des}\left(\sigma^{-1}\right)$, are contained in a fixed set $I \subseteq\{1,2, \ldots, n-1\}$. We can also apply the principle of inclusion-exclusion to sum over permutations in $\mathfrak{S}_{n}$ whose inverse descent sets are equal to $I$. Foata and Schützenberger (1978) were the first to give bijective proofs of these results in the $z=0$ case.

In Section 2, we introduce the insertion method, a technique due to Carlitz that gives a bijective proof of MacMahon's Theorem. Section 3 generalizes Carlitz's method to ordered multiset partitions, yielding a bijective proof of Theorem 1.1. We give an application to the theory of Macdonald polynomials in Section 4. 


\section{The Insertion Method}

\subsection{The Insertion Method for $\mathfrak{S}_{n}$}

One consequence of MacMahon's Equidistribution Theorem is a pair of recursions for the distributions of the inversion number and the major index over $\mathfrak{S}_{n}$ :

$$
D_{\mathfrak{S}_{n}}^{\text {inv }}(q)=[n]_{q} D_{\mathfrak{S}_{n-1}}^{\text {inv }}(q) \quad D_{\mathfrak{S}_{n}}^{\text {maj }}(q)=[n]_{q} D_{\mathfrak{S}_{n-1}}^{\text {maj }}(q) .
$$

On the other hand, these two statements imply MacMahon's result. Carlitz's insertion method gives bijective proofs of these statements which can be combined to build a recursive bijection $\psi_{n}: \mathfrak{S}_{n} \rightarrow \mathfrak{S}_{n}$ such that maj $(\psi(\sigma))=\operatorname{inv}(\sigma)$. We say that $\psi_{n}$ maps the inversion number to the major index. We outline Carlitz's insertion method below.

To prove the left statement in (1), one simply considers all the possible ways to insert $n$ into a permutation in $\mathfrak{S}_{n-1}$ to create a permutation in $\mathfrak{S}_{n}$. It is clear that, for $\sigma \in \mathfrak{S}_{n-1}$, inserting $n$ after the first $i$ elements of $\sigma$ creates $n-i-1$ new inversions and does not affect the previously existing inversions. For example, for $\sigma=5167324 \in \mathfrak{S}_{7}$, we can "label" these positions with subscripts that give the number of new inversions created by inserting an 8 at that position:

$$
{ }_{7} 5_{6} 1_{5} 6_{4} 7_{3} 3_{2} 2_{1} 4_{0} .
$$

This proves the inversion side of (1). The key to the insertion method is that something similar is true for the major index. In particular, we can label the spaces between elements of $\sigma \in \mathfrak{S}_{n-1}$, along with the left and right ends, according to the following scheme:

1. Label the position after $\sigma_{n-1}$ with a zero.

2. Label the descents of $\sigma$ right to left with $1,2, \ldots, \operatorname{des}(\sigma)$.

3. Label the position before $\sigma_{1}$ with $\operatorname{des}(\sigma)+1$.

4. Label the ascents of $\sigma$ from left to right with $\operatorname{des}(\sigma)+2, \ldots, n-1$.

For example, $\sigma=5167324$ receives the following labels in this setting:

$$
{ }_{4} 5_{3} 1_{5} 6_{6} 7_{2} 3_{1} 2{ }_{7} 4_{0}
$$

These labels give the change in major index that comes from inserting $n$ at that position; one proof of this fact is in Haglund et al. (2005b). This completes the proof of (1) and also gives a bijection that takes the inversion number to the major index. We include an example of this bijection in Figure 1 .

\subsection{The Insertion Method for $\mathfrak{S}_{A}$}

It is natural to hope that this proof can be extended to the permutations of any multiset $A$. That is, we would like to give insertion proofs that

$$
D_{\mathfrak{S}_{A}}^{\mathrm{inv}}(q)=\left[\begin{array}{l}
|A| \\
a_{n}
\end{array}\right]_{q} D_{\mathfrak{S}_{A^{\prime}}}^{\mathrm{inv}}(q) \quad D_{\mathfrak{S}_{A}}^{\mathrm{maj}}(q)=\left[\begin{array}{l}
|A| \\
a_{n}
\end{array}\right]_{q} D_{\mathfrak{S}_{A^{\prime}}}^{\mathrm{maj}}(q) .
$$




\begin{tabular}{llll}
$n$ & $\sigma$ & change in inv & $\psi_{n}(\sigma)$ \\
\hline 5 & 52143 & & 24153 \\
4 & 2143 & 4 & ${ }_{2} 2_{3} 4_{1} 1_{4} 3_{0}$ \\
3 & 213 & 1 & ${ }_{2} 2_{1} 1_{3} 3_{0}$ \\
2 & 21 & 0 & ${ }_{2} 2_{1} 1_{0}$ \\
1 & 1 & 1 & ${ }_{1} 1_{0}$
\end{tabular}

Fig. 1: To compute $\psi_{5}(52143)$, we remove the 5 and count the number of inversions lost by removing 5 . In this case, we have lost 4 inversions. We record this number in the third column and the resulting permutation in the $\sigma$ column. We repeat this process until we have reached $n=1$ and filled the first three columns of the table. To build our new permutation, we recursively place $n$ at the position that receives label $i$ in the major index labeling. (These labels have been italicized in the example.)

where $A^{\prime}=A \backslash\left\{n^{a_{n}}\right\}$. Such proofs would imply MacMahon's Equidistribution Theorem and provide a bijection between inversion number and major index.

The inversion side cooperates nicely. As before, inserting an $n$ to the right of $i$ elements of $\sigma \in \mathfrak{S}_{A}$ increases the inversion number by $\left|A^{\prime}\right|-i-1$. Hence this insertion can create between 0 and $\left|A^{\prime}\right|$ inversions. Furthermore, the position of a new $n$ has no affect on the number of inversions added by other $n$ 's; in other words, each insertion is independent of the other insertions. This allows us to compute $D_{\mathfrak{S}_{A}}^{\operatorname{inv}}(q)$.

$$
D_{\mathfrak{S}_{A}}^{\operatorname{inv}}(q)=\left.D_{\mathfrak{S}^{A^{\prime}}}^{\operatorname{inv}}(q)\left(\prod_{i=0}^{\left|A^{\prime}\right|} \frac{1}{1-q^{k} x}\right)\right|_{x^{a_{n}}}=\left[\begin{array}{c}
|A| \\
a_{n}
\end{array}\right]_{q} D_{\mathfrak{S}_{A^{\prime}}}^{\operatorname{inv}}(q)
$$

To prove the major index side of (2), we essentially recreate the bijection constructed in Foata and Han (2008) and Chen et al. (2010). Let $\left(\left(\begin{array}{l}S \\ k\end{array}\right)\right)$ denote the family of $k$-element multisets containing elements of the set $S$. We would like to establish a bijection

$$
\phi_{A}^{\mathrm{maj}}: \mathfrak{S}_{A^{\prime}} \times\left(\left(\begin{array}{c}
\left\{0,1, \ldots,\left|A^{\prime}\right|\right\} \\
a_{n}
\end{array}\right)\right) \rightarrow \mathfrak{S}_{A}
$$

such that

$$
\operatorname{maj}\left(\phi_{A}^{\mathrm{maj}}(\sigma, B)\right)=\operatorname{maj}(\sigma)+\sum_{b \in B} b .
$$

Such a map would provide a combinatorial proof of the major index side of (2). Before inserting any $n$ 's, we label $\sigma \in \mathfrak{S}_{A^{\prime}}$ in a manner reminiscent of Section 2.1.

1. Label the position after $\sigma_{\left|A^{\prime}\right|}$ with a zero.

2. Label the descents of $\sigma$ right to left with $1,2, \ldots, \operatorname{des}(\sigma)$.

3. Label the position before $\sigma_{1}$ with $\operatorname{des}(\sigma)+1$. 


\begin{tabular}{llll}
$A$ & $\sigma$ & $B$ & $\psi_{A}(\sigma)$ \\
\hline$\left\{1,2^{2}, 3,4^{3}\right\}$ & 2443214 & & 4432124 \\
& & & $44_{3} 3_{2} 2_{1} 12_{0}$ \\
& & & $4_{3} 3_{2} 2_{1} 12_{0}$ \\
$\left\{1,2^{2}, 3\right\}$ & 2321 & $\{3,3,0\}$ & ${ }_{3} 3_{2} 2_{1} 1_{4} 2_{0}$ \\
$\left\{1,2^{2}\right\}$ & 212 & $\{2\}$ & $2_{2} 2_{1} 1_{3} 2_{0}$ \\
& & & $2_{1} 1_{0}$ \\
$\left\{1,2^{2}\right\}$ & 1 & $\{1,0\}$ & $11_{0}$
\end{tabular}

Fig. 2: As in Section 2.1 we fill the first three columns of the table from top to bottom by removing all copies of the largest element and recording the multiset of inversions lost during each removal, which we call $B$. Then we fill the fourth column by using the labeling associated with the major index to repeatedly insert a new element at the position that received the largest remaining label in $B$.

4. Label the non-descents of $\sigma$ from left to right with $\operatorname{des}(\sigma)+2, \ldots,\left|A^{\prime}\right|$.

Write $B=\left\{b_{1} \geq b_{2} \geq \ldots \geq b_{a_{n}}\right\}$. We insert an $n$ into the position labeled $b_{1}$. Then we go through the labeling process again, stopping once we have used the label $b_{1}$. We insert an $n$ into the position labeled $b_{2}$. We repeat this process until we have processed each element of $B$. We omit the proof that this map satisfies the desired properties, opting instead to provide an example.

Let $A=\left\{1^{2}, 2,3^{3}, 4^{2}\right\}, \sigma=323113 \in \mathfrak{S}_{A^{\prime}}$, and $B=\left\{5^{2}\right\}$. We note that $\operatorname{maj}(\sigma)=4$. We begin by labeling $\sigma$ according to the labeling associated with the major index.

$$
{ }_{3} 3_{2} 2_{4} 3_{1} 1_{5} 1_{6} 3_{0} \text {. }
$$

We place a 4 at the label 5 to get 3231413 . We relabel this permutation, stopping when we use the label 5 .

$$
{ }_{4} 3_{3} 2_{5} 3_{2} 14_{1} 13_{0}
$$

Then we insert a 4 at the position labeled 5 to get 32431413 . As desired,

$$
\operatorname{maj}(32431413)=14=\operatorname{maj}(\sigma)+\sum_{b \in B} b=4+5+5 .
$$

Just as before, these insertion maps can be combined to yield a bijection $\psi_{A}: \mathfrak{S}_{A} \rightarrow \mathfrak{S}_{A}$ that takes inversion number to major index. We illustrate $\psi_{A}$ with the example in Figure 2

\section{A Generalization of the Insertion Method}

In order to prove Theorem 1.1, we define a superset of multiset permutations, which we will call descentstarred multiset permutations, in Section 3.1. These objects are in bijection with ordered multiset partitions. Next, we extend the inversion number and major index statistics to these new objects so that Theorem 1.1 is equivalent to the equidistribution of these statistics. Much of this preliminary material parallels work in Remmel and Wilson (2013). In Section 3.2, we sketch a proof of the equidistribution of these new statistics that generalizes the insertion method described in Section 2 This result is a new generalization of the main result of Remmel and Wilson (2013). Finally, we provide expressions for the distribution of these statistics in Section 3.3 


\subsection{Descent-Starred Multiset Permutations}

We consider the sets

$$
\begin{aligned}
\mathfrak{S}_{A, k}^{>} & =\left\{(\sigma, S): \sigma \in \mathfrak{S}_{A}, S \subseteq \operatorname{Des}(\sigma),|S|=k\right\} \\
\mathfrak{S}_{A}^{>} & =\bigcup_{k \geq 0} \mathfrak{S}_{A, k}^{>} .
\end{aligned}
$$

These are the descent-starred multiset permutations of the multiset $A$, since we can think of each position in $S$ as being followed by a star. For example, $4_{*} 3_{*} 1132_{*} 1 \in \mathfrak{S}_{\left\{1^{3}, 2,3^{2}, 4\right\}, 2}$. Notice that we do not need to star every descent. These objects can be seen as a particular way of recording ordered multiset partitions, where the stars indicate the adjacent elements that are combined into blocks. It will be more convenient in what follows to think of stars instead of blocks. We note that setting $q=1$ on either side of Theorem 1.1 gives

$$
\sum_{\sigma \in \mathfrak{S}_{A}}(1+z)^{\operatorname{des}(\sigma)}=\sum_{k \geq 0}\left|\mathfrak{S}_{A, k}^{>}\right| z^{k}
$$

so descent-starred multiset permutations are clearly relevant to Theorem 1.1 .

In order to prove Theorem 1.1, we need to establish statistics that correspond to each side of Theorem 1.1. We define the inversions of a descent-starred multiset permutation $(\sigma, S) \in \mathfrak{S}_{A}^{>}$by

$$
\operatorname{Inv}((\sigma, S))=\left\{(i, j): i<j, \sigma_{i}>\sigma_{j}, j \notin S,\{i, i+1, \ldots, j-1\} \nsubseteq S\right\}
$$

and the inversion number of $(\sigma, S)$ by

$$
\operatorname{inv}((\sigma, S))=|\operatorname{Inv}((\sigma, S))|=\operatorname{inv}(\sigma)-\sum_{i \in S} 1+\operatorname{inv}^{\square, i}(\sigma) .
$$

We set the major index of a descent-starred multiset permutation $(\sigma, S) \in \mathfrak{S}_{A}^{>}$to be

$$
\operatorname{maj}((\sigma, S))=\operatorname{maj}(\sigma)-\sum_{i \in S}|\operatorname{Des}(\sigma) \cap\{i, i+1, \ldots,|A|-1\}| .
$$

For example, let $(\sigma, S)=4_{*} 3_{*} 11322_{*}$. Then

$$
\begin{aligned}
\operatorname{Inv}((\sigma, S)) & =\{(1,4),(1,5),(1,7),(2,4),(2,7),(5,7)\} \\
\operatorname{inv}((\sigma, S)) & =5=12-1-2-4 \\
\operatorname{maj}((\sigma, S)) & =1+2+5+6-1-3-4=6 .
\end{aligned}
$$

These inversions have already received some attention when $A=\{1,2, \ldots, n\}$ in Steingrímmson (2007); Ishikawa et al. (2008). This major index first appeared in Remmel and Wilson (2013). Lemma 3.1 shows that our main theorem is equivalent to the equidistribution of the inversion number and major index over $\mathfrak{S}_{A, k}^{>}$. We outline a proof of this equidistribution in the next section. 


\section{Lemma 3.1}

$$
\begin{aligned}
& \sum_{k \geq 0} D_{\mathfrak{S}_{A, k}^{>}}^{\mathrm{inv}}(q) z^{k}=\sum_{\sigma \in \mathfrak{S}_{A}} q^{\operatorname{inv}(\sigma)} \prod_{i \in \operatorname{Des}(\sigma)}\left(1+\frac{z}{q^{1+\mathrm{inv}^{\square, i}(\sigma)}}\right) \\
& \sum_{k \geq 0} D_{\mathfrak{S}_{A, k}^{>} \mathrm{maj}}^{>}(q) z^{k}=\sum_{\sigma \in \mathfrak{S}_{A}} q^{\operatorname{maj}(\sigma)} \prod_{j=1}^{\operatorname{des}(\sigma)}\left(1+\frac{z}{q^{j}}\right)
\end{aligned}
$$

\subsection{Insertion Maps and a Bijective Proof of Theorem 1.1}

The final ingredient in proving Theorem 1.1 is to show that $D_{\mathfrak{S}_{A, k}^{>}}^{\text {inv }}(q)=D_{\mathfrak{S}_{A, k}^{>}}^{\text {maj }}(q)$. We will verify this statement by defining two classes of injections, which we will call insertion maps:

$$
\begin{aligned}
& \phi_{A, k, \ell}^{\text {inv }}: \mathfrak{S}_{A^{\prime}, \ell}^{>} \times\left(\begin{array}{c}
\left\{0,1, \ldots,\left|A^{\prime}\right|-\ell-1\right\} \\
k-\ell
\end{array}\right) \times\left(\left(\begin{array}{c}
\left\{0,1, \ldots,\left|A^{\prime}\right|-\ell\right\} \\
a_{n}-k+\ell
\end{array}\right)\right) \rightarrow \mathfrak{S}_{A, k}^{>} \\
& \phi_{A, k, \ell}^{\text {maj }}: \mathfrak{S}_{A^{\prime}, \ell}^{>} \times\left(\begin{array}{c}
\left\{0,1, \ldots,\left|A^{\prime}\right|-\ell-1\right\} \\
k-\ell
\end{array}\right) \times\left(\left(\begin{array}{c}
\left\{0,1, \ldots,\left|A^{\prime}\right|-\ell\right\} \\
a_{n}-k+\ell
\end{array}\right)\right) \rightarrow \mathfrak{S}_{A, k}^{>}
\end{aligned}
$$

When $k=0$ these maps will reduce to the insertion processes defined in Section 2.2. We can think of the element $U \in\left(\begin{array}{c}\left\{0,1, \ldots,\left|A^{\prime}\right|-\ell-1\right\} \\ k-\ell\end{array}\right)$ as providing the increases in the statistic that come from adding a new $n$ along with a new star, and the element $B \in\left(\left(\begin{array}{c}\left\{0,1, \ldots,\left|A^{\prime}\right|-\ell\right\} \\ a_{n}-k+\ell\end{array}\right)\right)$ providing the increases in the statistic that come from adding a new $n$ without a new star. These maps will cooperate with their respective statistics, i.e.

$$
\begin{aligned}
\operatorname{inv}\left(\phi_{A, k, \ell}^{\mathrm{inv}}((\sigma, S), U, B)\right) & =\operatorname{inv}((\sigma, S))+\sum_{u \in U} u+\sum_{b \in B} b \\
\operatorname{maj}\left(\phi_{A, k, \ell}^{\operatorname{maj}}((\sigma, S), U, B)\right) & =\operatorname{maj}((\sigma, S))+\sum_{u \in U} u+\sum_{b \in B} b .
\end{aligned}
$$

Then we will use these maps to form a bijection that sends inversion number to major index.

As usual, we discuss the inversion case first. Given $\sigma \in \mathfrak{S}_{A^{\prime}, \ell}^{>}$, we label the positions immediately after any $\sigma_{i}$ or before $\sigma_{1}$ from right to left with the numbers $0,1, \ldots,\left|A^{\prime}\right|-\ell$, skipping starred positions. Given $U \in\left(\begin{array}{c}\left\{0,1, \ldots,\left|A^{\prime}\right|-\ell-1\right\} \\ k-\ell\end{array}\right)$, let $U^{+}=\{u+1: u \in U\}$. We repeatedly remove the largest element in $U^{+} \cup B$, taking the element from $B$ if the largest elements are equal. Call this element $i$. If $i$ came from $B$, we place an $n$ at the position labeled $i$ and then relabel the sequence as before, stopping when we use label $i$. If $i$ came from $U^{+}$we insert a starred $n$ at the position labeled $i$ and then relabel, ending when we use the label $i-1$. We proceed in this manner, repeatedly taking $i$ to be the largest remaining element in $U^{+} \cup B$. We claim that this map is a bijection and satisfies the property 3 .

For example, say $A=\left\{1,2^{2}, 3^{2}, 4^{3}\right\}, k=3$, and $\ell=1$. We consider $(\sigma, S)=31223_{*} 1 \in \mathfrak{S}_{A^{\prime}, 1}^{>}$, $U=\{2,0\}$, and $B=\{3\}$. Then $U^{+}=\{3,1\}$ and $U^{+} \cup B=\{3,3,1\}$. We label $(\sigma, S)$ as follows:

$$
{ }_{5} 3_{4} 1_{3} 2_{2} 2_{1} 3_{*} 1_{0}
$$


The largest element in $U^{+} \cup B$ is 3, so we insert a 4 at the position labeled 3. Since we took this element from $B$, we do not add a new star. Then we label this new descent-starred multiset permutation, using only labels up to 3 .

$$
314_{3} 2_{2} 2_{1} 3_{*} 1_{0}
$$

The largest remaining element of $U^{+} \cup B$ is still 3, so we put a 4 at the position labeled 3. Since this element comes from $U^{+}$, we star this new 4. Labeling once more, we get

$$
3144_{*} 2_{2} 2_{1} 3_{*} 1_{0}
$$

Finally, we insert a starred 4 at the position labeled 1 to get $3144_{*} 224_{*} 3_{*} 1$. We check that this new permutation has the desired inversion number.

$$
\begin{aligned}
& \operatorname{inv}\left(3 \quad 144_{*} 224_{*} 3_{*} 1\right)=17-1-1-4 \\
& =11=\operatorname{inv}\left(\begin{array}{lllll}
3 & 1 & 2 & 2 & 3 * 1
\end{array}\right)+2+3
\end{aligned}
$$

To define $\phi_{A, k, \ell}^{\mathrm{maj}}$, we form $U^{+}$as before. Our labeling changes to reflect the change in statistic. We label the unstarred positions of $\sigma$ as in Section 2 Specifically, we label the unstarred descents from right to left, then the non-descents from left to right with increasing labels $0,1, \ldots,\left|A^{\prime}\right|-\ell$. We repeatedly remove the largest element $i$ from $U^{+} \cup B$, taking $i$ from $B$ if the largest elements are equal. Then we proceed through the following sequence:

1. Insert an $n$ at the position labeled $i$.

2. Move each star that to the right of the new $n$ one descent to the left.

3. If $i$ came from $U^{+}$, star the rightmost descent.

4. Relabel as before, stopping at the label $i$ if $i$ came from $B$ and $i-1$ if $i$ came from $U^{+}$.

We claim that this process gives a bijection and satisfies (4). For example, let us again consider $A=$ $\left\{1,2^{2}, 3^{2}, 4^{3}\right\}, k=3$, and $\ell=1$ with $(\sigma, S)=31223_{*} 1 \in \mathfrak{S}_{A^{\prime}, 1}^{>}, U=\{2,0\}$, and $B=\{3\}$. We label $(\sigma, S)$ with the labeling associated with major index to obtain

$$
{ }_{2} 3_{1} 1_{3} 2_{4} 2_{5} 3_{*} 1_{0} .
$$

We insert a 4 at the position labeled 3 and then move all stars to the right of that position one spot to their left. Since we took 3 from $B$, we do not star the rightmost descent, resulting in $314_{*} 2231$. We label this object as follows.

$$
{ }_{3} 3{ }_{2} 14_{*} 223{ }_{1} 1_{0}
$$

Again we choose the position labeled 3. This time we star the rightmost descent after shifting stars because 3 came from $U^{+}$, yielding $43_{*} 14223_{*}$. Finally we label this element

$$
4_{2} 3_{*} 14_{1} 223_{*} 1_{0}
$$




\begin{tabular}{llllll}
$A$ & $k$ & $(\sigma, S)$ & $U$ & $M$ & $\psi_{A, k}((\sigma, S))$ \\
\hline$\left\{1^{3}, 2,3^{2}, 4\right\}$ & 3 & $4_{*} 3_{*} 1132_{*} 1$ & & & $3_{*} 2_{*} 11431$ \\
$\left\{1^{3}, 2,3^{2}\right\}$ & 2 & $3_{*} 1132_{*} 1$ & $\{4\}$ & $\emptyset$ & ${ }_{2} 3_{*} 2_{*} 1_{3} 1_{4} 3_{1} 1_{0}$ \\
& & & & & ${ }_{2} 2_{*} 1_{2} 13_{*} 1_{0}$ \\
$\left\{1^{3}, 2\right\}$ & 1 & $112_{*} 1$ & $\{2\}$ & $\{1\}$ & $12_{*} 1_{2} 1_{3} 1_{0}$ \\
$\left\{1^{3}\right\}$ & 0 & 111 & $\{0\}$ & $\emptyset$ & $1_{2} 1_{2} 1_{3} 1_{0}$
\end{tabular}

Fig. 3: We repeatedly remove all of the largest elements (and their stars) from the starred permutation and recording the number of inversions lost in the $U$ and $B$ columns. Starred elements contribute to the $U$ column and unstarred elements contribute to the $B$ column. Once we have reached the final row, we use this information to build the $\psi_{A, k}((\sigma, S))$ column from bottom to top. We use the elements of $U^{+} \cup M$ to select the positions at which to insert new largest elements. This insertion follows the procedure laid out in the definition of $\phi^{\text {maj }}$.

We insert a 4 at the position labeled 1, shift stars, and star the rightmost descent to get $43_{*} 144_{*} 223_{*} 1$. We check that this new permutation has the desired major index.

$$
\begin{aligned}
\operatorname{maj}\left(43_{*} 144_{*} 2\right. & \left.23_{*} 1\right) \\
& =1+2+5+8-1-2-3 \\
& =10=\operatorname{maj}\left(31223_{*} 1\right)+2+3 .
\end{aligned}
$$

These two insertion maps work together to provide a bijection $\psi_{A, k}: \mathfrak{S}_{A, k}^{>} \rightarrow \mathfrak{S}_{A, k}^{>}$that takes inversion number to major index. This bijection, along with Lemma 3.1. proves Theorem 1.1, We work through an example of this bijection in Figure 3

There are a number of other consequences of this method. For example, it is not difficult to show that our bijection, like Carlitz's bijection, preserves left-to-right maxima. This property turns out to be important in the full proof of Theorem 4.1 .

We can also apply the method to other statistics. Rawlings (1981) defined a family of statistics called the $r$-major index which depend on a positive integer $r$. The $r$-major index includes the inversion number and the usual major index as special cases. We can prove an analog of Theorem 1.1 for this whole family of statistics. Such extensions will be proved in the full version of this paper.

\subsection{Distribution}

At this point it is natural to wonder if we can describe the distributions of our statistics on $\mathfrak{S}_{A, k}^{>}$. Define

$$
M_{A, k}(q)=D_{\mathfrak{S}_{A, k}^{>}}^{\mathrm{inv}}(q)=D_{\mathfrak{S}_{A, k}^{>}}^{\mathrm{maj}}(q) .
$$

We know from MacMahon's Theorem that $M_{A, 0}(q)=\left[\begin{array}{c}|A| \\ a_{1}, \ldots, a_{n}\end{array}\right]_{q}$. In general, we can only give a recursive description of $M_{A, k}(q)$. Applying standard $q$-binomial identities to the insertion maps given above, we see

$$
M_{A, k}(q)=\sum_{\ell=0}^{k} q^{\left(\begin{array}{c}
k-\ell \\
2
\end{array}\right)}\left[\begin{array}{c}
|A|-\ell \\
k-\ell
\end{array}\right]_{q}\left[\begin{array}{c}
|A|-k \\
a_{n}-k+\ell
\end{array}\right]_{q} M_{A^{\prime}, \ell}(q)
$$


with suitable initial conditions. We can rearrange the terms of the last sum to obtain the identity

$$
M_{A, k}(q)=\sum_{\ell=0}^{k} q^{\left(\begin{array}{c}
k-\ell \\
2
\end{array}\right)}\left[\begin{array}{c}
|A|-\ell \\
a_{n}
\end{array}\right]_{q}\left[\begin{array}{c}
a_{n} \\
k-\ell
\end{array}\right]_{q} M_{A^{\prime}, \ell}(q)
$$

We could also sum to $a_{n}$ instead of $k$.

We can obtain another expression for this polynomial in the special case $a_{1}=\ldots=a_{n}=a$. Before we can state this expression, we must define a $q$-analog of the (generalized) Stirling numbers of the second kind. The $q=1$ case of these polynomials was studied in Blasiak et al. (2003). We define these polynomials recursively by

$$
S_{n, k}^{(a)}(q)=\sum_{i=0}^{a}\left[\begin{array}{c}
i \\
a-(k-i)
\end{array}\right]_{q} \frac{[a]_{q} !}{[k-i]_{q} !} S_{n-1, i}^{(a)}(q) .
$$

Proposition 3.2 If $A=\left\{1^{a}, 2^{a}, \ldots, n^{a}\right\}$ for some $a \in \mathbb{N}$, then

$$
M_{A, k}(q)=\frac{[a n-k]_{q} !}{\left([a]_{q} !\right)^{n}} S_{n, a n-k}^{(a)}(q) .
$$

The proof follows from (5) and (6). There is also a more combinatorial proof of this fact that relies on certain rook placements; we will include this proof in the full version of this paper.

\section{Starred Macdonald Polynomials}

In this section we develop an application of our work thus far to the theory of symmetric functions. We insert our inv and maj statistics into the combinatorial definition of Macdonald polynomials for hook shapes, as given (for any shape) in Haglund et al. (2005a). This yields functions whose coefficients are four-variable polynomials instead of the usual two-variable polynomial coefficients. Our work in Section 3 allows us to prove that these polynomials are symmetric and to expand them into Schur functions. Readers unfamiliar with symmetric function theory or Macdonald polynomials are encouraged to reference Stanley (1999) and Haglund et al. (2005a), respectively.

For convenience, let $[a, b]=\{a, a+1, \ldots, b\}$ and, for any statistic stat, let

$$
\operatorname{stat}_{[a, b]}(\sigma)=\operatorname{stat}\left(\sigma_{a} \sigma_{a+1} \ldots \sigma_{b}\right) .
$$

We will also need two new statistics, one on multiset permutations and one on descent-starred multiset permutations:

$$
\begin{aligned}
\operatorname{rlmaj}(\sigma) & =\sum_{i \in \operatorname{Des}(\sigma)}(n-i) \\
\operatorname{rlmaj}((\sigma, S)) & =\operatorname{rlmaj}(\sigma)-\sum_{i \in S}|\operatorname{Des}(\sigma) \cap[1, i]| .
\end{aligned}
$$

We set $\widetilde{H}_{n, k}(x ; q, t, u, v)$ equal to

$$
\sum_{\sigma} x^{\sigma} q^{\operatorname{inv}_{[k+1, n]}(\sigma)} t^{\operatorname{maj}_{[1, k]}(\sigma)} \prod_{i \in \operatorname{Des}(\sigma) \cap[k+1, n]}\left(1+u q^{-\operatorname{inv}_{[k+1, n]}^{\square, i}(\sigma)-1}\right) \prod_{j=1}^{|\operatorname{Des}(\sigma) \cap[1, k]|}\left(1+v t^{-j}\right)
$$


where the sum is over words of length $n$ in the alphabet $\mathbb{N}$. We will refer to these polynomials as starred Macdonald polynomials of hook shape. The motivation for these polynomials is to consider writing a descent-starred multiset permutation $(\sigma, S)$ in the Young diagram for the shape $\left(n-k, 1^{k}\right)$. Calculating our maj and inv statistics down the column and across the row, respectively, and summing over all such descent-starred multiset permutations yields the definition given above. When $u=v=0$, we obtain the (modified) Macdonald polynomial for the shape $\left(n-k, 1^{k}\right)$, as proven in Haglund et al. (2005a) and discussed further in Adin et al. (2008).

Our main result in this section allows us to transfer many important properties of the Macdonald polynomials to the starred Macdonald polynomials of hook shape. The $u=v=0$ case of this result was originally proven by Stembridge (1994). The result requires some definitions on standard Young tableaux, which are defined, among other places, in Stanley (1999). We write SYT $(\lambda)$ for the collection of standard Young tableaux of shape $\lambda$. In particular, the descent set of a standard Young tableau $T$ (in French notation) is the set of all $i$ such that $i+1$ is strictly north (and weakly west) of $i$ in $T$. Then, for any standard Young tableau $T$ with $n$ entries,

$$
\operatorname{maj}(T)=\sum_{i \in \operatorname{Des}(T)} i \quad \operatorname{rlmaj}(T)=\sum_{i \in \operatorname{Des}(T)}(n-i)
$$

Theorem 4.1 The starred Macdonald polynomials of hook shape are symmetric. Furthermore, for $\lambda \vdash n$ the coefficient of the Schur function $s_{\lambda}(x)$ in $\widetilde{H}_{n, k}(x ; q, t, u, v)$ is equal to

$$
\sum_{T \in \operatorname{SYT}(\lambda)} q^{\operatorname{rlmaj}_{[k+1, n]}(T)} t^{\operatorname{maj}_{[1, k]}(T)} \prod_{i=1}^{|\operatorname{Des}(T) \cap[k+1, n]|}\left(1+u q^{-i}\right) \prod_{j=1}^{|\operatorname{Des}(T) \cap[1, k]|}\left(1+v t^{-j}\right)
$$

The proof is similar to the proof of Theorem 3 in Haglund (2004), except we fill the shape with descentstarred multiset permutations instead of multiset permutations. Where Haglund (2004) uses Foata's bijection on multiset permutations, we use our bijection $\psi_{A, k}$ on descent-starred multiset permutations, as developed in Section 3.2 .

To conclude, we mention two ways in which we hope to extend this work in the future. There are deep connections between Macdonald polynomials and representation theory, many of which involve the Garsia-Haiman module, as defined in Garsia and Haiman(1993). Adin et al. (2008) gave several bases for the Garsia-Haiman module in the hook case. One of their bases naturally transforms into a basis which corresponds to the starred Macdonald polynomials of hook shape. An interesting question is whether or not there exists a definition of the linear span of this basis as the linear span of partial derivatives of a certain determinant, as there is in the classical case due to Garsia-Haiman modules.

In a different direction, we would like to define and explore starred Macdonald polynomials for nonhook shapes. The full combinatorial formulation of Macdonald polynomials in Haglund et al. (2005a) essentially allows one to either star horizontally or vertically, giving analogs of Macdonald polynomials with three-variable polynomials for coefficients. At this point, we have been unable to define a fourvariable generalization in the non-hook case that retains desirable properties such as symmetry. Such a development has the potential to further our understanding of the theory of Macdonald polynomials. 


\section{References}

R. Adin, J. Remmel, and Y. Roichman. The combinatorics of the Garsia-Haiman modules for hook shapes. Electronic Journal of Combinatorics, 15, 2008.

P. Blasiak, K. Penson, and A. Solomon. The general boson normal ordering problem. Physics Letters A, 309:198-205, 2003.

L. Carlitz. A combinatorial property of $q$-Eulerian numbers. Amer. Math. Monthly, 82:51-54, 1975.

W. Y. Chen, S. Poznanovik, C. H. Yan, and A. L. Yang. Major index for 01-fillings of moon polyominoes. Journal of Combinatorial Theory, 117(8):1058-1081, 2010.

D. Foata. On the Netto inversion number of a sequence. Proc. Amer. Math. Soc., 19:236-240, 1968.

D. Foata and G.-N. Han. Fix-mahonian calculus, i: Two transformations. European Journal of Combinatorics, 29(7):1721-1732, 2008.

D. Foata and M. Schützenberger. Major index and inversion number of permutations. Math. Nachr., 83: 143-159, 1978.

A. M. Garsia and M. Haiman. A graded repesentation model for the Macdonald polynomials. Proc. Nat. Acad. Sci., 90:3607-3610, 1993.

J. Haglund. A combinatorial model for the Macdonald polynomials. Proc. Nat. Acad. Sci. USA, 101: 16127-16131, 2004.

J. Haglund, M. Haiman, and N. Loehr. A combinatorial formula for Macdonald polynomials. Journal of the American Mathematical Society, 18:735-761, 2005a.

J. Haglund, N. Loehr, and J. Remmel. Statistics on wreath products, perfect matchings, and signed words. Eur. J. Combin., 26:835-868, 2005 b.

M. Ishikawa, A. Kasraoui, and J. Zeng. Euler-Mahonian statistics on ordered set partitions. SIAM Journal of Discrete Mathematics, 22:1105-1137, 2008.

P. A. MacMahon. Combinatory Analysis, volume 1. Cambridge University Press, 1915.

D. Rawlings. The $r$-major index. J. Combin. Theory Ser. A, 115(2):175-183, 1981.

J. Remmel and A. Wilson. An extension of MacMahon's equidistribution theorem to ordered set partitions. Preprint available at http://sites.google.com/site/andywilsonmath/ research, 2013.

R. P. Stanley. Enumerative Combinatorics, volume 2. Cambridge University Press, 1999.

E. Steingrímmson. Statistics on ordered partitions of sets. arXiv:math/0605670v4, April 2007.

J. R. Stembridge. Some particular entries of the two-pararemeter Kostka matrix. Proc. Amer. Math. Soc., 121:469-490, 1994. 